\title{
Depth Profiling Magnetic Structure Using Scanning Electron Microscopy with Polarization Analysis (SEMPA)
}

\author{
B. J. McMorran, D. T. Pierce, and J. Unguris
}

Center for Nanoscale Science and Technology, National Institute of Standards and Technology, Gaithersburg, MD 20899

We demonstrated a new magnetic depth profiling technique using scanning electron microscopy with polarization analysis (SEMPA) [1]. SEMPA records quantitative images of surface magnetization (Fig. 1) by measuring the spin polarization of secondary electrons. We recently developed a surface removal technique for cleaning samples using $50 \mathrm{eV}$ Ar ion sputtering that leaves the underlying magnetization largely intact [2]. Here we show that this surface removal technique can, under certain circumstances, be combined with SEMPA to reveal the 3D magnetization inside a sample.

As a proof of concept, we studied the magnetization in a $\mathrm{Co} / \mathrm{Pd}$ multilayer, a material notable for its strong perpendicular magnetic anisotropy. These materials are magnetically "hard" and have no flux closure domains near the top surface - key properties that enable depth profile analysis in this case. The sample we studied had graded magnetic anisotropy; the deposition conditions were tuned such that the magnetization varied in three regions of the multilayer stack, labeled here as Regions A, B, and C. A schematic of the sample structure is illustrated in Fig. 2(a) [3].

We recorded a series of SEMPA images (similar to Fig. 1) as we sputtered away all of the material of this structure. A profile of the magnetization angle distribution as a function of sputter depth is shown in Fig. 2(b). This plot was extracted from the SEMPA image series by binning the recorded magnetization vectors according to their angle in the $x-z$ plane ( $\mathbf{z}$ is normal to film surface). In the figure, red corresponds to a large number of magnetization vectors pointing in a particular direction. The vertical stripes at $90^{\circ}$ and $270^{\circ}$ confirm that the magnetization points primarily in the up $(+\mathbf{z})$ and down (-z) directions. In the top $25 \%$ of the multilayer structure, in Region A, there is a preponderance of down domains, but as we sputter into Region B there is a sharp increase in the area of up domains. When Region $\mathrm{C}$ is revealed by the milling process, there is yet another transition in the magnetization, such that up and down magnetic domains have an equal distribution. Finally, after most of the magnetic material has been milled away there is a sharp transition to in-plane magnetization, as shown at the bottom of the plot. This in-plane transition could be due to a mixed Co-Pd layer that forms at the top surface during the sputtering process, which appears to adopt the underlying magnetization of the stack through most of the sputtering process. [4]

References

[1] M. R. Scheinfein, J. Unguris, M. H. Kelley, D. T. Pierce, and R. J. Celotta, Rev. Sci. Instrum. 61 (1990) 2501.

[2] B. J. McMorran et al., J. Appl. Phys. accepted for publication May 2010.

[3] This sample is equivalent to the "3P" multilayer in B. J. Kirby et al., J. Appl. Phys. 105 (2009)

07 C929.

[4] The authors are grateful to Randy Dumas and Kai Liu for the Co/Pd samples. 


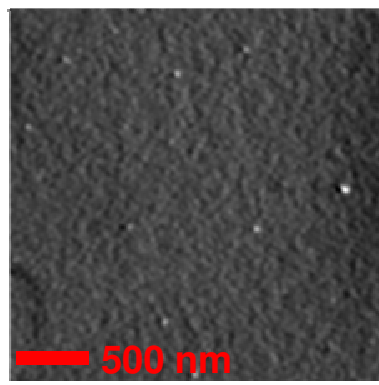

(a) intensity

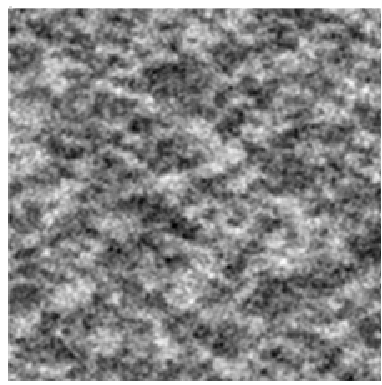

(b)

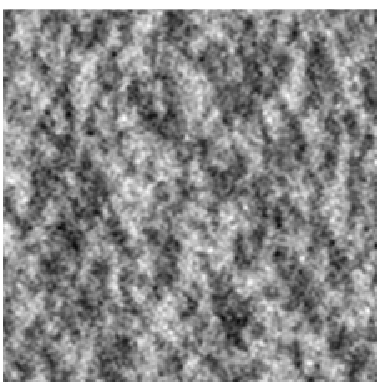

(c)

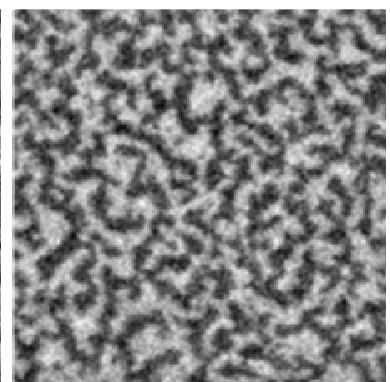

(d)

$M_{z}$

FIG. 1. SEMPA images of a magnetic multilayer. A single SEMPA scan simultaneously records (a) surface topography (similar to a standard SEM image), and two vector components of the surface magnetization, either the in-plane components, $\mathrm{M}_{\mathrm{x}}(\mathrm{b})$ and $\mathrm{M}_{\mathrm{y}}(\mathrm{c})$, or one in-plane and one perpendicular component, $\mathrm{M}_{\mathrm{x}}$ and $\mathrm{M}_{\mathrm{z}}(\mathrm{d})$. Two consecutive scans are required to collect images of all three components of surface magnetization, as shown here.

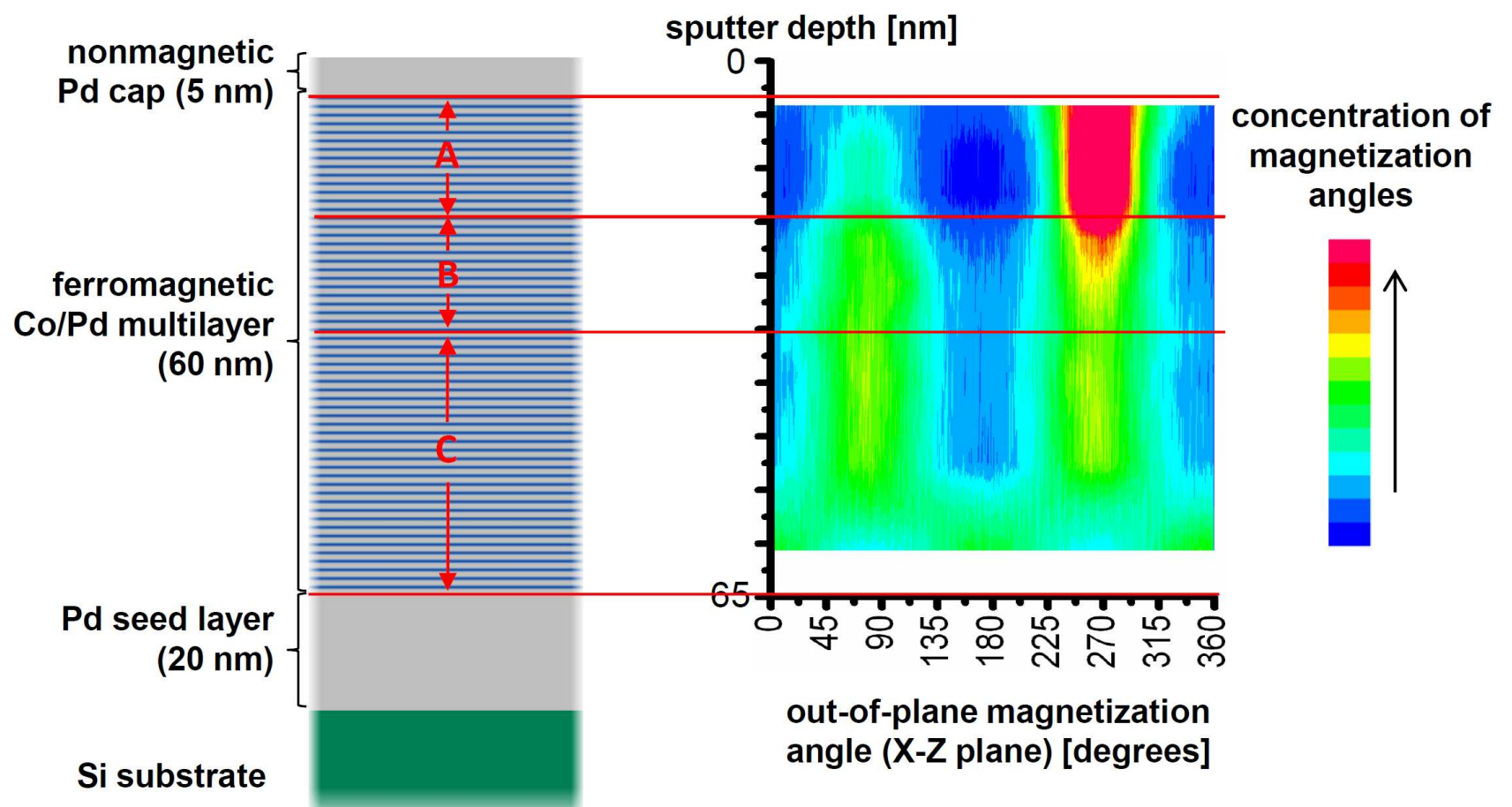

(a)

(b)

FIG. 2. (a) The compositional structure of the $\mathrm{Co} / \mathrm{Pd}$ multilayers, $\operatorname{Pd}(5 \mathrm{~nm})[\mathrm{Co}(0.4 \mathrm{~nm}) / \mathrm{Pd}(0.6$ $\mathrm{nm}]_{60} / \mathrm{Pd}(20 \mathrm{~nm})$, is shown on the left. By design, the magnetization of the multilayers varies in the vertical direction, such that Regions "A", "B", and "C" each have unique magnetic properties. (b) Plots of magnetization angle concentration, as a function of depth in the sample. Red areas correspond to a large tendency for magnetization to align to a preferred direction. 